\title{
Prevalencia de disfunción diastólica de acuerdo con las guías diagnósticas de 2009 y 2016. Relación con la valoración hemodinámica no invasiva
}

\author{
Prevalence of diastolic dysfunction (DD) according to the diagnostic guidelines of 2009 \\ and 2016. Relationship with non-invasive hemodynamic assessment
}

\author{
Adrián Carlessi*, Leonel Perello, Evangelina Garello y Carlos Valli \\ Servicio de Cardiología, Departamento de Ecocardiografía, Sanatorio San Gerónimo. Santa Fe, Argentina
}

\begin{abstract}
Resumen
Objetivos: Evaluar la prevalencia de DD de acuerdo con los criterios de las guías del 2009 y 2016, y su relación con la distensibilidad arterial sistémica (DAS) y la resistencia vascular periférica (RVP). Material y métodos: Se analizó a 306 pacientes $\geq$ 40 años de edad, con fracción de expulsión $\geq 50 \%$. Se calcularon en todos DAS y RVP. Resultados: La prevalencia de DD fue de $32.7 \%$ y 22.9\% según las guías de 2009 y 2016, respectivamente ( $p=<0.0001)$. De acuerdo con las guías del 2009 , los pacientes con $D D$ presentaron una media de DAS menor que aquéllos con función normal $(p=0.0001)$, de modo similar a las guías del 2016 ( $p=0.0007$ ). La DD, según las guías de 2009 y 2016, mostró valores más altos de RVP que los normales ( $p=0.005$ y $p=0.018$, respectivamente). Asimismo, la DD fue predictora, en el análisis univariado, de $D A S<0.60 \mathrm{ml} . \mathrm{mm} \mathrm{Hg}^{1}$ y RVP > 1,400 mmHg.min..$^{-1}$ según ambas guías. En el análisis multivariado, la DD, de acuerdo con las guías del 2009, persistió como predictor independiente de RVP > 1,400 mmHg.min. $\mathrm{F}^{-1}$. Conclusión: Las guías del 2016 reducen la prevalencia de DD. Ésta, tanto en las guías del 2009 como en las del 2106, fue predictor univariado de DAS $<0.60 \mathrm{ml}^{\mathrm{mmHg}} \mathrm{m}^{-1} \mathrm{RVP}>1,400 \mathrm{mmHg}$. min. $I^{-1}$. La DD, de acuerdo con las guías de 2009, resultó predictora independiente de $R V P>1,400 \mathrm{mmHg} \cdot \mathrm{min}^{-1} \mathrm{I}^{-1}$.
\end{abstract}

Palabras clave: Diástole. Hemodinamia. Doppler. Ecocardiografía.

\begin{abstract}
Objectives: To assess the prevalence of developmental disabilities (DD) according to the criteria of the 2009 and 2016 guidelines, and its association with systemic arterial compliance (DAS) and peripheral vascular resistance (RVP). Material and methods: 306 patients aged $\geq 40$ years, with ejection fraction $\geq 50 \%$ were analyzed. It was estimated in all DAS and RVP. Results: The prevalence of DD was $32.7 \%$ and $22.9 \%$ according to the 2009 and 2016 guidelines, respectively ( $p \leq 0.0001$ ). Patients with DD according to the 2009 guideline had a lower average of DAS than those with normal function ( $p=0.0001)$. Similar with the 2016 guide $(p=0.0007)$. The presence of $D D$ according to the 2009 and 2016 guideline showed higher RVP values than normal values ( $p=0.005$ and $p=0.018$, respectively). The $D D$ according to both guidelines was a predictor, in the univariate analysis, of $D A S<0.60 \mathrm{ml} . \mathrm{mmHg}^{-1}$ and RVP $>1400 \mathrm{mmHg} \cdot \mathrm{min} . \mathrm{I}^{-1}$. The DD
\end{abstract}

Correspondencia:

*Adrián Carlessi

E-mail: adriancarlessi@gmail.com

DOI: 10.24875/ACM.20000340
Disponible en internet: $23-12-2020$ Arch Cardiol Mex. 2020;90(4):420-426 www.archivoscardiologia.com 1405-9940 / @ 2020 Instituto Nacional de Cardiología Ignacio Chávez. Publicado por Permanyer. Este es un artículo open access bajo la licencia CC BY-NC-ND (http://creativecommons.org/licenses/by-nc-nd/4.0/). 
according to the 2009 guideline persisted as an independent predictor, in the multivariate analysis, of RVP > $1400 \mathrm{mmHg}$. min. $I^{-1}$. Conclusion: The 2016 guide decreases the prevalence of DD. The DD, both from the 2009 and 2106 guidelines, were univariate predictors of DAS $<0.60 \mathrm{ml} . \mathrm{mmHg}^{-1}$ and RVP $>1400 \mathrm{mmHg} \cdot \mathrm{min}^{-l^{-1}}$. The DD according to the 2009 guide, was an independent predictor of RVP $>1400 \mathrm{mmHg} \cdot \mathrm{min} . \mathrm{I}^{-1}$.

Key words: Diastole. Hemodynamics. Doppler. Echocardiography.

\section{Introducción}

La diástole es el período del ciclo cardíaco cuya afectación y consiguiente disfunción son críticas y necesarias para el desarrollo de insuficiencia cardíaca, en especial en los pacientes con función sistólica conservada ${ }^{1}$, lo cual tiene implicaciones para el pronósti$\mathrm{co}^{2}$. El estudio de la función diastólica de forma no invasiva, a través de la ecocardiografía, ha evolucionado en el tiempo, y los valores y parámetros utilizados han variado, de acuerdo con las guías de expertos. En el año 2016 se publicaron las recomendaciones de la American Society of Echocardiography (ASE) y la European Association of Cardiovascular Imaging (EACVI) ${ }^{3}$; estas guías hicieron énfasis en la simplificación de los parámetros a utilizar y se basaron en los más factibles y reproducibles. Además, se diferenciaban de las recomendaciones del año 2009 de la ASE y la European Association of Echocardiography (EAE) ${ }^{4}$, que utilizan algoritmos y parámetros más complejos.

La valoración no invasiva de parámetros hemodinámicos, a través de la determinación de la distensibilidad arterial sistémica (DAS) y la resistencia vascular periférica (RVS), demostró utilidad en la evaluación y el pronóstico de pacientes con afección valvular aórti$\mathrm{ca}^{5,6}$. Este trabajo compara la prevalencia de disfunción diastólica (DD) de acuerdo con los criterios de estas dos guías y su relación con la DAS y la RVP, parámetros que indican afectación hemodinámica y podrían establecer el pronóstico, calculados en forma no invasiva.

\section{Material y métodos}

\section{Población}

Se incluyó en forma consecutiva a 306 pacientes de 40 o más años de edad derivados al centro de los autores para la realización de ecocardiograma Doppler, y que presentaran fracción de expulsión $(\mathrm{FE}) \geq 50 \%$. Se excluyó del estudio a los pacientes con antecedentes de infarto agudo de miocardio, taquicardia sinusal (frecuencia cardíaca > 110 latidos por minutos), fibrilación o aleteo auricular en el momento del estudio, marcapasos definitivo implantado o enfermedad valvular cardíaca mayor de leve.

\section{Evaluación ecocardiográfica}

Todos los estudios ecocardiográficos se efectuaron con el equipo de ultrasonido Philips Afinity 50. La cuantificación de cámaras y función se realizaron según los lineamientos de las recomendaciones de ASE/EACVI del año 2015. Los tipos de hipertrofia ventricular se clasificaron de acuerdo con las nuevas indicaciones de la EACVI ${ }^{8}$.

Los parámetros de disfunción diastólica se cuantificaron en las vistas apicales. Las velocidades de llenado mitral (picos E y A) y el tiempo de desaceleración del pico $E$ (TDE) se determinaron con el Doppler pulsado, a nivel de los extremos distales de los velos mitrales, en la apertura de la válvula mitral (con promedio de tres latidos consecutivos). Mediante Doppler tisular se calcularon las velocidades del anillo mitral lateral y septal (e' y a') y se realizaron las correspondientes determinaciones de relaciones ( $E / A$ y $\left.E / e^{\prime}\right)$.

La medición de la velocidad de regurgitación tricuspídea se obtuvo a través de Doppler continuo y, con el uso de la ecuación simplificada de Bernoulli, se estableció el gradiente de presión ventriculoauricular derecho.

\section{Mediciones hemodinámicas no invasivas}

Todos los pacientes fueron objeto de medición no invasiva con esfigmomanómetro de la presión arterial durante el procedimiento. Se calculó la presión de pulso (PP) a través de la operación: presión arterial sistólica menos presión arterial diastólica. La presión arterial media (PAM) se determinó mediante el producto de la presión arterial sistólica por el tercio de la PP. Se realizó el cálculo de la DAS con la siguiente ecuación":

$$
\text { DAS = volumen sistólico indexado/(PP) }
$$

La RVP se estableció a través de la siguiente fórmula10:

$$
\mathrm{RVP}=80 \times \mathrm{PAM} / \text { gasto cardíaco }
$$




\section{Clasificación de la disfunción diastólica}

Para determinar la DD a través de las guías del 2009 de ASE/EAE se tomaron en primera instancia los valores de e' lateral y septal (> 10 y 8 respectivamente), junto con el volumen de la aurícula izquierda $\left(>34 \mathrm{ml} / \mathrm{m}^{2}\right)$; cuando estaban presentes estos parámetros se tomaron en cuenta los siguientes parámetros para determinar el grado de disfunción diastólica: relación E/A, TDE y relación e/e' promedio. Fue necesario tener los parámetros positivos para establecer el grado de disfunción diastólica y, cuando los parámetros eran ambiguos, se consideró el grado de disfunción diastólica como indeterminado.

Para establecer DD a través de las guías del 2016 de ASE/EACVI se utilizaron cuatro parámetros: a) relación E/e' promedio > 14; b) relación e' septal $<70$ relación e' lateral $<10 ; c)$ volumen auricular izquierdo $>34 \mathrm{ml} / \mathrm{m}^{2} ;$ d) velocidad de regurgitación tricuspídea $>2.8 \mathrm{~m} / \mathrm{s}$. Se necesitaron más del $50 \%$ de los parámetros positivos, que fueron posibles obtener, para determinar la presencia de DD. Si era positivo, la mitad de los parámetros obtenidos se consideró como indeterminado. Para la determinación del grado de DD se usaron los siguientes parámetros: relación E/A y la velocidad pico $E$. Con posterioridad se tomaron en cuenta: a) volumen auricular izquierdo $>34 \mathrm{ml} / \mathrm{m}^{2}$; b) velocidad de regurgitación tricuspídea $>2.8 \mathrm{~m} / \mathrm{s}$; c) relación E/e' promedio $>14$. Si dos de estos últimos parámetros eran positivos, se determinó el grado de DD; en caso de disponer de sólo dos criterios, debían ser ambos positivos. En caso de ser uno positivo y otro negativo se consideró el grado de DD como indeterminado.

\section{Análisis estadístico}

Las variables continuas se presentan como media \pm desviación estándar (DE) y las variables categóricas como porcentajes. La concordancia entre las dos clasificaciones se determinó con el coeficiente kappa. Las comparaciones entre individuos normales y DD con las variables hemodinámicas se efectuaron mediante la prueba de Rank Sum Test; y la comparación entre la función normal, indeterminada o disfunción diastólica de las guías del 2016 de ASE/EACVI con las medidas hemodinámicas se efectuó a través del análisis de varianza unidireccional o prueba de Kruskal-Wallis para variables continuas. La prueba chi cuadrada o la prueba exacta de Fisher, según correspondiera, se emplearon para determinar diferencias de variables categóricas.
Tabla 1. Características clínicas y ecocardiográficas de la población

\begin{tabular}{|c|c|}
\hline Variable & Valor \\
\hline Edad (años) & $59.6( \pm 10.4)$ \\
\hline Sexo femenino (\%) & 59.2 \\
\hline Hipertensión arterial (\%) & 63.4 \\
\hline Diabetes $(\%)$ & 16.3 \\
\hline Dislipemia (\%) & 34.3 \\
\hline Índice de masa corporal & $29.6( \pm 5.59)$ \\
\hline Obesidad (\%) & 39.9 \\
\hline $\mathrm{FE}(\%)$ & $65.2( \pm 5.59)$ \\
\hline Volumen auricular izquierdo $\left(\mathrm{ml} / \mathrm{m}^{2}\right)$ & $35.6( \pm 8.02)$ \\
\hline Índice de masa miocárdica $\left(\mathrm{g} / \mathrm{m}^{2}\right)$ & $102( \pm 29.02)$ \\
\hline Volumen al final de la diástole (ml) & $85.4( \pm 23)$ \\
\hline Volumen al final de la sístole (ml) & $30.9( \pm 10.5)$ \\
\hline Hipertrofia excéntrica (\%) & 41.8 \\
\hline Hipertrofia concéntrica (\%) & 10.8 \\
\hline Relación E/A & $0.95( \pm 0.34)$ \\
\hline TDE (ms) & $222( \pm 49.9)$ \\
\hline Relación E/e' promedio & $8.62( \pm 2.9)$ \\
\hline Velocidad de regurgitación tricuspídea $(\mathrm{m} / \mathrm{s})$ & $2.32( \pm 0.34)$ \\
\hline Presión arterial sistólica $(\mathrm{mmHg})$ & $131.38( \pm 18.87)$ \\
\hline Presión arterial diastólica (mmHg) & $73.43( \pm 10.1)$ \\
\hline DAS (ml.mmHg-1) & $0.73( \pm 0.23)$ \\
\hline $\operatorname{RVP}\left(\mathrm{mmHg} \cdot \min . \mathrm{I}^{-1}\right)$ & $1,436.53( \pm 352.81)$ \\
\hline
\end{tabular}

Las variables dicotómicas que fueron estadísticamente significativas para la predicción de DAS $<0.60 \mathrm{ml}$. $\mathrm{mmHg}^{-1}$ y RVP $>1,400 \mathrm{mmHg} \cdot \mathrm{min}^{-\mathrm{I}^{-1}}$ se sometieron a la prueba de regresión logística, para un análisis multivariado. Se consideraron estadísticamente significativos valores de $p=<0.05$. Estos análisis estadísticos se efectuaron con los programas STATICTIX 8.0, EPI INFO 6.0 $0^{\mathrm{TM}}$ y MedCalc.

\section{Resultados}

En este estudio se incluyó a 306 pacientes, de los cuales $59.2 \%$ fue del sexo femenino, con media de edad de 59.6 ( \pm 10.4) (Tabla 1). El 85.6\% de los pacientes no presentaba síntomas, el $2 \%$ tenía disnea de clase funcional (CF) I de la New York Heart 


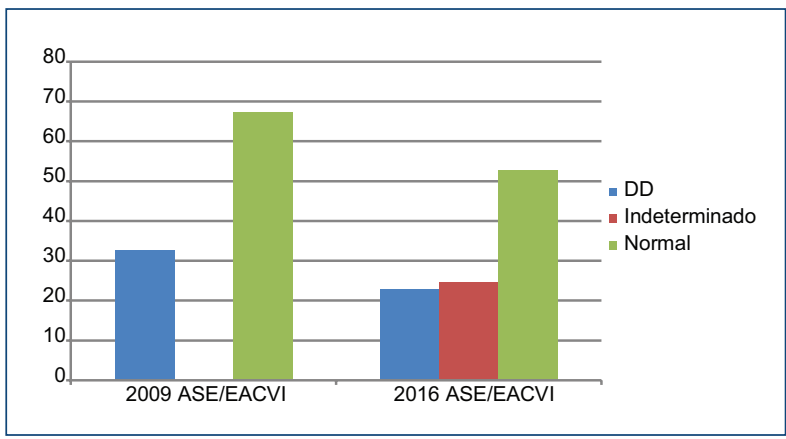

Figura 1. Prevalencia de disfunción diastólica según las diferentes guías.

Association, $11.6 \%$ con CF II y $0.6 \%$ con CF III. De los pacientes hipertensos, el $60.5 \%$ se hallaba bajo medicación antihipertensiva. De éstos, el $51.8 \%$ estaba tratado con un fármaco antihipertensivo, el $41 \%$ con dos y el $7.2 \%$ con tres o más. La factibilidad de obtener los cuatro parámetros que determinan la presencia de DD en las guías del 2016 de ASE/EACVI fue del $59.5 \%$, la de tres parámetros de $40.2 \%$ y en $0.3 \%$ sólo se obtuvieron dos parámetros. Los parámetros que evalúan la DD en las guías del 2009 de ASE/EAE se obtuvieron en el $99.7 \%$. No fue posible la determinación de la velocidad de regurgitación tricuspídea en el $41.2 \%$ de los casos.

La prevalencia de DD, según las guías del 2009 de ASE/EAE, fue del $32.7 \%$, mientras que del $22.9 \%$ (Fig. 1) de acuerdo con el algoritmo de las guías del 2016 de ASE/EACVI. En los pacientes con DD, según las guías del 2009 de ASE/EAE, los grados fueron: I, 42\%; II, $30 \%$; III, 0\%; e indeterminado, 28\%. Cuando se clasificó la DD de acuerdo con las guías del 2016 de ASE/EACVI los grados fueron: I, 17.1\%; II, 44.3\%; III, $1,4 \%$; e indeterminado, $37.2 \%$.

La concordancia entre los criterios de DD de ambas guías fue moderado (coeficiente kappa, 0.56); de los 100 pacientes con criterios de DD a partir de las guías del 2009 de ASE/EAE, 42 se reclasificaron sin DD con los criterios de las guías del 2016 de ASE/EACVI (Tabla 2). De estos 42 pacientes, 40 (95\%) se reclasificaron como indeterminados y $2(5 \%)$ con función diastólica normal.

La media de DAS en los sujetos con DD, según las guías del 2009 de ASE/EAE, fue de 0.67 ( \pm 0.22) $\mathrm{ml} . \mathrm{mmHg}^{-1} \mathrm{y}$ en aquéllos con función diastólica normal fue de $0.76( \pm 0.20) \mathrm{ml} \cdot \mathrm{mmHg}^{-1}(p=0.0001)$. Los pacientes con DD, de acuerdo con las guías del 2016 de ASE/EA$\mathrm{CVI}$, tuvieron DAS media de $0.67( \pm 0.22) \mathrm{ml}_{\mathrm{mmHg}}{ }^{-1}$, mientras que los que presentaban función diastólica
Tabla 2. Concordancia entre los criterios de DD (coeficiente kappa, 0.56; intervalo de confianza, $0.46-0.66 ; p=0.051$ )

\begin{tabular}{|l|c|c|c|}
\hline & \multicolumn{2}{|c|}{$\begin{array}{c}\text { Disfunción diastólica 2009 } \\
\text { ASE/EACVI }\end{array}$} & \\
\hline $\begin{array}{l}\text { Disfunción diastólica } \\
2016 \text { ASE/EACVI }\end{array}$ & Sí & No & \\
\hline Sí & 58 & 12 & $70(22.9 \%)$ \\
\hline No & 42 & 194 & $236(77.1 \%)$ \\
\hline & $100(32.7 \%)$ & $206(67.3 \%)$ & 306 \\
\hline
\end{tabular}

normal era de $0.75( \pm 0.24) \mathrm{ml} . \mathrm{mmHg}^{-1}(p=0.0007)$. La media de RVP de los pacientes con función diastólica normal, según las guías del 2009 de ASE/EAE, fue de 1,403 $( \pm 338)$ mmHg.min..$^{-1}$ y con DD de 1,509 $( \pm 366)$ mmHg.min..$^{-1}(p=0.005)$. En los individuos con función diastólica conservada, según las guías del 2016 de ASE/EACVI, la RVP media fue de 1,412 ( \pm 334$) \mathrm{mmHg}$. min. $I^{-1}$ y en aquéllos con DD dicho parámetro fue de $1,523( \pm 390)$ mmHg.min..$^{-1}(p=0.018)$.

Con base en las guías del 2016 de ASE/EACVI, los pacientes indeterminados presentaron media de DAS menor que los sujetos con función diastólica normal (0.73 ml. $\mathrm{mmHg}^{-1}$ vs. $0.76 \mathrm{ml} . \mathrm{mmHg}^{-1}$, respectivamente) y mejor perfil respecto de los pacientes con DD ( $p=0.005$ para los tres grupos). Igual comportamiento presentó la RVP, la cual fue de $1,516 \mathrm{mmHg} \cdot \mathrm{min}^{-\mathrm{I}^{-1}}$ para los pacientes con DD, $1,478.41 \mathrm{mmHg} \cdot \mathrm{min}^{-l^{-1}}$ para los individuos indeterminados y $1,385 \mathrm{mmHg} \cdot \mathrm{min}^{-l^{-1}}$ para los enfermos con función diastólica normal $(p=0.009)$.

Entre los pacientes con criterios suficientes para DD, según ambas guías, se encontró un mayor número de sujetos con RVP $>1,400$ mmHg.min..$^{-1}$ y DAS $<0.60 \mathrm{ml}$. $\mathrm{mmHg}^{-1}$ que entre los pacientes $\sin$ criterios suficientes para DD (Fig. 2).

Resultaron variables predictoras de DAS $<0.60 \mathrm{ml}$. $\mathrm{mmHg}^{-1}$, hipertensión arterial $(\mathrm{HTA})(\mathrm{p}=<0.0001)$, dislipemia $(p=0.017)$, obesidad ( $p=0.015)$, tabaquismo $(p=0.015)$, la DD según las guías del 2009 de ASE/EAE ( $p=0.003)$ y la DD según las guías del 2016 de ASE/EACVI $(p=0.009)$. En el análisis multivariado, la HTA persiste como única variable predictora de DAS $<0.60$ ml. $\mathrm{mmHg}^{-1}(p=0.0034)$ (Tabla 3).

Las variables predictoras de RVP $>1,400 \mathrm{mmHg}$. min..$^{-1}$ fueron HTA $(p=0.001)$, diabetes (DBT) $(p=0.045)$, DD según las guías del 2009 de ASE/EAE $(p=0.0008)$ y DD según las guías del 2016 de ASE/EACVI $(p=0.013)$. En el análisis de regresión 


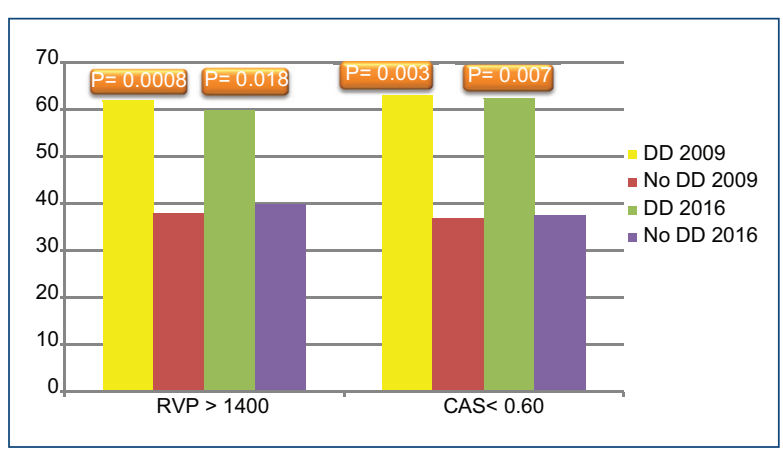

Figura 2. Prevalencia de RVP $>1,400 \mathrm{mmHg} \cdot \min . \mathrm{I}^{-1}$ y DAS $<0.60 \mathrm{mmHg} \cdot \mathrm{min}^{-1} \mathrm{I}^{-1}$ según la presencia 0 ausencia de DD.

Tabla 3. Regresión logística de variables predictoras de DAS $<0.60$ ml. $\mathrm{mmHg}^{-1}$

\begin{tabular}{|l|c|c|c|}
\hline Variables & Coeficiente & Error estándar & $\mathbf{P}$ \\
\hline Constante & -2.30540 & 0.32239 & 0.0000 \\
\hline Dislipemia & 0.31155 & 0.28479 & 0.2740 \\
\hline Obesidad & 0.44915 & 0.27653 & 0.1043 \\
\hline HTA & 0.96627 & 0.32952 & 0.0034 \\
\hline Tabaquismo & 0.62080 & 0.40682 & 0.1270 \\
\hline 2009 ASE/EACVI & 0.48730 & 0.34743 & 0.1607 \\
\hline 2016 ASE/EACVI & 0.17517 & 0.37606 & 0.6414 \\
\hline
\end{tabular}

Tabla 4. Regresión logística de variables predictoras de RVP > 1,400 mmHg.min..$^{-1}$

\begin{tabular}{|l|c|c|c|}
\hline Variables & Coeficiente & Error estándar & $\mathbf{P}$ \\
\hline Constante & -0.58261 & 0.20984 & 0.0055 \\
\hline HTA & 0.63987 & 0.25188 & 0.0111 \\
\hline 2009 ASE/EACVI & 0.69768 & 0.31411 & 0.0263 \\
\hline 2016 ASE/EACVI & 0.07684 & 0.35175 & 0.8271 \\
\hline DBT & -0.68164 & 0.33102 & 0.0395 \\
\hline
\end{tabular}

logística persisten como variables predictoras la DBT $(p=0.0395)$, HTA $(p=0.0111)$ y la DD según las guías del 2009 de ASE/EAE ( $p=0.0263)$ (Tabla 4).

\section{Discusión}

La evaluación de la función diastólica a través de la ecocardiografía Doppler comienza con la medición de las velocidades del flujo transmitral a nivel de los extremos distales de los velos mitrales y sus cambios con la edad, la normalidad y los diferentes trastornos ${ }^{11}$. La marcada dependencia de las condiciones de carga hizo necesario contar con otros parámetros que reflejaran en forma más precisa el grado de compromiso diastólico y la determinación de las presiones de llenado ${ }^{12}$. La velocidad de excursión del anillo mitral se agregó al estudio de la función diastólica y su cociente con la velocidad del flujo transmitral ${ }^{13}$. Estos parámetros junto con la medición del volumen auricular izquierdo y la velocidad de regurgitación tricuspídea emergieron como los parámetros de evaluación medulares de la función diastólica. Sus criterios de utilización variaron desde la complejidad de las guías del 2009 de ASE/EAE hasta un intento de simplificación de las guías del 2016 de ASE/EACVI.

En este trabajo se identificó una disminución significativa ( $p=<0.0001$ ) de la prevalencia de DD según los criterios del 2009 de ASE/EAE (32.7\%) respecto de los cálculos de las guías del 2016 de ASE/EACVI (22.9\%). Esta disminución de la prevalencia, a pesar de ser estadísticamente significativa, es de menor cuantía que la publicada en el trabajo de Almeida, et al. ${ }^{14}$, en el cual la prevalencia de las guías del 2009 de ASE/ EAE fue del $38.1 \%$ y la del 2016 de ASE/EACVI de $1.4 \%$. En el estudio de los autores, la concordancia entre ambas guías fue moderada (coeficiente kappa $=0.56)$, diferente al citado estudio, en el que la concordancia fue pobre entre ambas guías (coeficiente kappa $=0.18$ ). Resulta notorio que en el estudio de Almeida, et al., el porcentaje de factibilidad de obtener los cuatros parámetros de las guías del 2016 de ASE/EACVI fue del $98.4 \%$ y en el estudio de los autores fue del 59.5\%; la velocidad de regurgitación tricuspídea fue la variable menos factible de obtener $(58.8 \%$ de los pacientes). Este trabajo concuerda con estudios poblacionales de valoración de la presión arterial pulmonar sistólica, en los que la factibilidad de sus cálculos, a través de la medición de la regurgitación tricuspídea, es del 62 a $69 \% 15,16$.

Por otro lado, en un estudio reciente se observó ${ }^{17}$ en pacientes con sepsis que las guías del 2016 de ASE/ EACVI identificaban mayor prevalencia e incidencia en el seguimiento de DD que las guías del 2009 de ASE/ $E A E$, sin diferencias tan marcadas tanto en la prevalencia como en la incidencia de DD entre ambas guías, como sucede en el estudio de los autores, aunque con resultados antagónicos; esto es probable en el contexto clínico diferente en el que se realizó la valoración de la diástole. 
Los factores de riesgo cardiovasculares producen menor capacidad de la pared arterial para adaptarse a los volúmenes y las presiones que se transmiten desde el ventrículo izquierdo y aumentan la resistencia (poscarga) periférica a la cual se debe enfrentar, con la consiguiente elevación de las presiones de llenado ventricular izquierdo para mantener el gasto cardíaco. Los parámetros hemodinámicos no invasivos (DAS y RVP), que reflejan esta afectación, mostraron valores alterados en los pacientes con DD de ambas guías estudiadas, sin diferencias sustanciales entre ambas. La presencia de DD, de acuerdo con las dos guías, fueron predictoras de DAS $<0.60 \mathrm{ml} \cdot \mathrm{mmHg}^{-1}$ y de RVP $>1,400 \mathrm{mmHg} \cdot \mathrm{min}^{-\mathrm{I}^{-1}}$, en el análisis univariado. Sólo la DD establecida por las guías del 2009 de ASE/EAE fue predictora independiente de $\mathrm{RVP}>1,400 \mathrm{mmHg} \cdot \mathrm{min} . \mathrm{I}^{-1}$. Los autores identificaron diferencias en los perfiles hemodinámicos de los pacientes catalogados como normales, indeterminados o DD de acuerdo con las guías de 2016, lo que revela que los individuos considerados como indeterminados tienen un perfil hemodinámico peor que los pacientes normales $\mathrm{y}$ un mejor estado hemodinámico, no invasivo, respecto de aquéllos con disfunción diastólica. Estudios recientes mostraron la capacidad de las guías del 2016, en cuanto a la sensibilidad y especificidad, para predecir la elevación de las presiones de llenado, evaluadas en forma invasiva ${ }^{18}$; en este estudio, la capacidad de predicción entre ambas guías no fue sustancialmente diferente. Esto es similar al estudio de los autores en el que ambas clasificaciones mostraron capacidad predictora para la presencia de alteraciones hemodinámicas, medidas en forma no invasiva, con una discreta superioridad de las guías del 2009 de ASE/EAE, ya que esta última resultó predictora independiente de RVP $>1,400 \mathrm{mmHg} \cdot \mathrm{min}^{-\mathrm{I}^{-1}}$.

\section{Conclusión}

Las guías del 2016 de ASE/EACVI reducen la prevalencia de disfunción diastólica en relación con las guías del 2009 de ASE/EAE. Ambas guías predicen alteración en la DAS y RVP. La potencia predictiva de sufrir alteraciones hemodinámicas, a través de la medición no invasiva de la DAS y RVP, es ligeramente superior en las guías del 2009 de ASE/EAE en comparación con las del 2016.

\section{Financiamiento}

La presente investigación no recibió ninguna beca específica de agencias de los sectores público, comercial, o sin ánimo de lucro.

\section{Conflicto de intereses}

Los autores declaran no tener ningún conflicto de intereses.

\section{Responsabilidades éticas}

Protección de personas y animales. Los autores declaran que para esta investigación no se han realizado experimentos en seres humanos ni en animales.

Confidencialidad de los datos. Los autores declaran que en este artículo no aparecen datos de pacientes.

Derecho a la privacidad y consentimiento informado. Los autores declaran que en este artículo no aparecen datos de pacientes.

\section{Bibliografía}

1. Wan SH, Vogel MW, Chen HH. Pre-clinical diastolic dysfunction. J Am Coll Cardiol. 2014;63:407-16

2. Nagueh SF. Prognostic power of mitral annulus indices of left ventricular diastolic function. J Am Heart Assoc. 2014;3:e001012.

3. Nagueh SF, Smiseth OA, Appleton CP, Byrd BF $3^{\text {rd }}$, Dokainish H, Edvardsen $\mathrm{T}$, et al. Recommendations for the evaluation of left ventricular diastolic function by echocardiography: an update from the American Society of Echocardiography and the European Association of Cardiovascular Imaging. Eur Heart J Cardiovasc Imaging. 2016;17:1321-60.

4. Nagueh SF, Appleton CP, Gillebert TC, Marino PN, Oh JK, Smiseth OA, et al. Recommendations for the evaluation of left ventricular diastolic function by echocardiography. Eur J Echocardiogr. 2008;10:165-93.

5. Briand M, Dumesnil JG, Kadem L, Tongue AG, Rieu R, Garcia D, Pibarot $P$. Reduced systemic arterial compliance impacts significantly LV afterload and functions in aortic stenosis: implications for diagnosis and treatment. J Am Coll Cardiol. 2005;46:291-298.

6. Hachicha Z, Dumesnil JG, Bogaty P, Pibarot P. Paradoxical low flow, low gradient severe aortic stenosis despite preserved ejection fraction is associated with higher afterload and reduced survival. Circulation. 2007;115:2856-2864.

7. Lang R, Badano L, Mor-Avi V, Afilalo J, Armstrong A, Ernand L, et al. Recommendations for cardiac chamber quantification by echocardiography in adults: an update from the American Society of Echocardiography and the European Association of Cardiovascular Imaging. J Am Soc Echocardiogr. 2015;28:1-39.

8. Marwick TH, Gillebert TC, Aurigemma G, Chirinos J, Derumeaux G, Galderisi M, et al. Recommendations on the use of echocardiography in adult hypertension: a report from the European Association of Cardiovascular Imaging (EACVI) and the American Society of Echocardiography (ASE). J Am Soc Echocardiogr. 2015;28:727-54

9. Chemla D, Hébert J-L, Coirault C. Total arterial compliance estimated by stroke volume-to-aortic pulse pressure ratio in humans. Am J Physiol Heart Circ Physiol. 1998;274:H500-5.

10. Kadem L, Dumesnil JG, Rieu R, Durand LG, García D, Pibarot P. Impact of systemic hypertension on the assessment of aortic stenosis. Heart. 2005:91:354-361.

11. Kuo LC, Quinones MA, Rokey R, Sartori M, Abinader EG, Zoghbi WA. Quantification of atrial contribution to left ventricular filling by pulsed wave Doppler echocardiography and the effect of age in normal and diseased hearts. Am J Cardiol. 1987;59:1174-8.

12. Hurrell DG, Nishimura RA, Ilstrup DM, Appeton CP. Utility of preload alteration in assessment of left ventricular filling pressure by Doppler echocardiography: a simultaneous catheterization d Doppler echocardiographic study. J Am Coll Cardiol. 1997;30:459-67.

13. Ommen SR, Nishimura RA, Appleton CP, Miller FA, Oh JK, Redfield MM, et al. Clinical utility of Doppler echocardiography and tissue Doppler imaging in the estimation of left ventricular filling pressures: a comparative simultaneous Doppler-catheterization study. Circulation. 2000;102:1788-94.

14. Almeida JG, Fontes-Carvalho $R$, Sampaio $F$, Ribeiro J, Bettencourt $P$ Flachskampf FA, et al. Impact of the 2016 ASE/EACVI recommendations on the prevalence of diastolic dysfunction in the general population. Eur Heart J Cardiovasc Imaging. 2018;19:380-6 
Arch Cardiol Mex. 2020;90(4)

15. Brittain E, Nwabuo C, Xu M, Gupta D, Hemnes A, Moreira H, et al. Echocardiographic pulmonary artery systolic pressure in the Coronary Artery Risk Development in Young Adults (CARDIA) Study: associations with race and metabolic dysregulation. J Am Heart Assoc. 2017;6:e005111. DOI:10.1161/JAHA.116.005111.

16. Lam C, Borlaug B, Kane G, Enders F, Rodeheffer R, Redfield M. Age-associated increases in pulmonary artery systolic pressure in the general population. Circulation. 2009;119:2663-2670.

17. Clancy D, Scully T, Slama M, Huang S, McLean A, Sam R. Application of updated guidelines on diastolic dysfunction in patients with severe sepsis and septic shock. Ann Intensive Care. 2017; 7:121-130

18. Balaney B, Medvedofsky D, Mediratta A, Singh A, Ciszek B, Kruse E, et al. Invasive validation of the echocardiographic assessment of left ventricular filling pressures using the 2016 diastolic Guidelines: head-tohead comparison with the 2009 Guidelines. J Am Soc Echocardiogr. 2018;31:79-88. 